\title{
Neutrino evolution in dense matter and electromagnetic field
}

\author{
A. V. Chukhnova ${ }^{1, *}$ and A. E. Lobanov ${ }^{1, * *}$ \\ ${ }^{1}$ Moscow State University, Faculty of Physics, Department of theoretical physics
}

\begin{abstract}
Neutrino evolution in dense matter and electromagnetic field is studied within quantum-field theoretical description on the base of a modification of the Standard Model, where the neutrinos are combined in $S U(3)$-multiplets. A quantum wave equation for neutrino in matter and electromagnetic field is obtained. In quasi-classical approximation a general method for calculating the probabilities of different spin-flavor transitions of neutrino in constant homogeneous field and moving matter with constant polarization is developed. In two-flavor model the explicit form of the solutions is obtained in constant electromagnetic field taking into account the transition magnetic moments. The obtained spin-flavor transition probabilities are compared to the results for unpolarized moving matter.
\end{abstract}

\section{Introduction}

Oscillations of ultra-relativistic neutrinos in vacuum are well-described within the phenomenological theory of neutrino oscillations, based on pioneer papers [1, 2] (see also [3]). The interaction with the medium is taken into account with the help of an effective potential [4]. This interaction results in modification of the phenomenological formulas for neutrino oscillations. Within the approach it is possible to predict the resonance behavior of the transition probabilities, which is called Mikheev-Smirnov-Wolfenstein effect [5].

However, as neutrino is a massive fermion, a complete description of neutrino evolution must include the information about the evolution of neutrino spin. Such description can be constructed on the base of the modification of the Standard model, proposed in [6, 7]. In this modification all the fermions are combined in $S U(3)$-multiplets. One-particle wave functions are elements of the representation space of the direct product of Poincare group and $S U(3)$ group. This helps to overcome the difficulties in constructing the Fock space for the neutrino, which are discussed, e.g., in [8]. Within this approach the Fock space for neutrino flavor states can be constructed, and so we can describe the processes of neutrino production and oscillations on the base of the S-matrix formalism. If we properly define the initial and the final states of the system, then the dependence of the probabilities of different processes on the distance between the source and the detector arises. For high energy neutrinos this dependence is in agreement with the phenomenological formulas for neutrino oscillations (for more details see [7]).

\footnotetext{
*e-mail: av.chukhnova@physics.msu.ru

**e-mail: lobanov@phys.msu.ru
} 
In the framework of this approach, wave functions $\Psi(x)$ describe the neutrino multiplet as a whole. These wave functions are 12-component objects, obeying the modified Dirac equation

$$
\left(\mathrm{i} \gamma^{\mu} \partial_{\mu} \mathbb{I}-\mathrm{M}\right) \Psi(x)=0 .
$$

In this equation $\mathbb{I}$ is the $3 \times 3$ identity matrix, and $\mathrm{M}$ is a Hermitian mass matrix of the neutrino multiplet, which can be represented as follows

$$
\mathrm{M}=\sum_{l=1}^{3} m_{l} \mathbb{P}_{l} .
$$

Here the eigenvalues $m_{l}$ of the mass matrix can be identified with the masses of the multiplet components, and the matrices $\mathbb{P}_{l}$ are orthogonal projectors on the subspaces with these masses. In Eq. (1) the product of the Dirac matrices and the matrices $\mathbb{M}, \mathbb{I}$ is defined as the tensor product.

The interaction with the medium may be taken into account on the base of the DiracSchwinger equation (see, e.g. [9]) with the help of the effective potentials, which arise due to reduction of the neutrino mass operator in matter. The potential

$$
f^{\alpha(e)}=\sqrt{2} G_{\mathrm{F}}\left(j^{\alpha(e)}-\lambda^{\alpha(e)}\right)
$$

determines the neutrino interaction with the electrons via charged currents, and the potential

$$
f^{\alpha(\mathrm{N})}=\sqrt{2} G_{\mathrm{F}} \sum_{i=e, p, n}\left(j^{\alpha(i)}\left(T^{(i)}-2 Q^{(i)} \sin ^{2} \theta_{\mathrm{W}}\right)-\lambda^{\alpha(i)} T^{(i)}\right)
$$

determines the neutral current contribution. For the derivation of the neutrino wave equation in matter see [10].

Since the mass states are well-defined, the interaction with the electromagnetic field may be taken into account with the use of the Pauli terms, and the neutrino wave equation is as follows [11]

$$
\begin{aligned}
\left(\mathrm{i} \gamma^{\mu} \partial_{\mu} \mathbb{I}-\mathrm{M}-\frac{1}{2} \gamma^{\alpha} f_{\alpha}^{(e)}(1\right. & \left.+\gamma^{5}\right) \mathrm{P}^{(e)}-\frac{1}{2} \gamma_{\alpha} f^{\alpha(\mathrm{N})}\left(1+\gamma^{5}\right) \mathbb{I} \\
& \left.-\frac{\mathrm{i}}{2} \mu_{0} F^{\mu \nu} \sigma_{\mu \nu} \mathrm{M}-\frac{\mathrm{i}}{2} F^{\mu v} \sigma_{\mu \nu} \mathrm{M}_{h}-\frac{\mathrm{i}}{2}{ }^{\star} F^{\mu v} \sigma_{\mu \nu} \mathrm{M}_{a h}\right) \Psi(x)=0 .
\end{aligned}
$$

Here $\mathbb{P}^{(e)}$ is the projector on the neutrino state with electron flavor, $F^{\mu v}$ is the electromagnetic field tensor, ${ }^{\star} F^{\mu \nu}=-\frac{1}{2} e^{\mu \nu \rho \lambda} F_{\rho \lambda}$ is the dual electromagnetic field tensor. The diagonal magnetic moments $\mu^{(l)}$ are given by the expression

$$
\mu^{(l)}=m_{l} \mu_{0}
$$

where $\mu_{0}$ is defined by the relation

$$
\mu_{0}=\frac{3 e G_{\mathrm{F}}}{8 \sqrt{2} \pi^{2}}
$$

The interaction with transition magnetic and electric moments $\mu^{(i j)}, \varepsilon^{(i j)}$ is taken into account by introducing the Hermitian and the anti-Hermitian matrices of transition moments $\mathbb{M}_{h}$ and $\mathrm{M}_{a h}$ (see, e.g., [12]). In the mass representation these matrices take the form

$$
\mathrm{M}_{h}=\frac{1}{2}\left(\begin{array}{ccc}
0 & \left(m_{1}+m_{2}\right) k_{12} & \left(m_{1}+m_{3}\right) k_{13} \\
\left(m_{2}+m_{1}\right) k_{21} & 0 & \left(m_{2}+m_{3}\right) k_{23} \\
\left(m_{3}+m_{1}\right) k_{31} & \left(m_{3}+m_{2}\right) k_{32} & 0
\end{array}\right)
$$




$$
\mathbf{M}_{a h}=\frac{1}{2}\left(\begin{array}{ccc}
0 & \left(m_{1}-m_{2}\right) k_{12} & \left(m_{1}-m_{3}\right) k_{13} \\
\left(m_{2}-m_{1}\right) k_{21} & 0 & \left(m_{2}-m_{3}\right) k_{23} \\
\left(m_{3}-m_{1}\right) k_{31} & \left(m_{3}-m_{2}\right) k_{32} & 0
\end{array}\right)
$$

where

$$
k_{i j}=\frac{\mu_{0}}{2} \sum_{l=e, \mu, \tau} \mathbb{U}_{l i}^{*} \mathbb{U}_{l j} \frac{M_{l}^{2}}{M_{W}^{2}} .
$$

The matrices $\mathbb{M}_{h}, \mathbb{M}_{a h}$ in the flavor representation may be obtained with the use of the mixing matrix $\mathbb{U}$.

\section{Quasi-classical approximation}

The range of energies, for which Eq. (5) is valid, is bounded from above. However, we can study the behavior of neutrinos of arbitrary low energies, including the relic neutrinos. Here we will consider only ultra-relativistic neutrinos, when the neutrino energies satisfy the condition $m_{l}^{2} / \mathcal{E}_{v}^{2} \ll 1$, that is when the phenomenological formulas for neutrino oscillations are also valid.

In this case we can use the quasi-classical approximation to describe the neutrino evolution. Since for the ultra-relativistic particles de Broglie wavelength is small, we can interpret $x^{\mu}$ not as the coordinates of the event space, but as the coordinates of the neutrino. If we consider the neutrino multiplet moving with a constant 4-velocity $u^{\mu}\left(u^{2}=1\right)$, then we can make the substitution $x^{\mu}=\tau u^{\mu}$. It means that the neutrino evolution is characterized by its proper time $\tau$ only. The proper time is related to the path length $L$ as follows

$$
\tau=L /|\mathbf{u}| \text {. }
$$

By analogy with quasi-classical spin wave functions [13], we introduce quasi-classical spin-flavor wave functions $\Psi(\tau)$, which describe spin-flavor coherent neutrino states. The corresponding evolution equation follows from Eq. (5), if we make the substitution

$$
\gamma^{\mu} \partial_{\mu} \Rightarrow \gamma^{\mu}\left(\frac{\partial \tau}{\partial x^{\mu}}\right) \frac{d}{d \tau}=\gamma^{\mu} u_{\mu} \frac{d}{d \tau} .
$$

It should be noted that substitution (12) is possible only when $u^{\mu}=$ const. Since the quasiclassical spin-flavor wave functions are required to satisfy the condition $\gamma^{\mu} u_{\mu} \Psi(\tau)=\Psi(\tau)$, the evolution equation takes the form

$$
\left(\mathrm{iII} \frac{d}{d \tau}-\mathcal{F}\right) \Psi(\tau)=0
$$

where

$$
\begin{aligned}
& \mathcal{F}=\mathrm{M}+\frac{1}{2}\left(f^{(e)} u\right) \mathrm{P}^{(e)}+\frac{1}{2}\left(f^{(\mathrm{N})} u\right)+ \frac{1}{2} R_{e} \mathrm{P}^{(e)} \gamma^{5} \gamma^{\sigma} s_{\sigma}^{(e)} \gamma^{\mu} u_{\mu}+\frac{1}{2} R_{\mathrm{N}} \gamma^{5} \gamma^{\sigma} s_{\sigma}^{(\mathrm{N})} \gamma^{\mu} u_{\mu} \\
&-\mu_{0} \mathrm{M} \gamma^{5} \gamma^{\mu \star} F_{\mu \nu} u^{\nu}-\mathrm{M}_{h} \gamma^{5} \gamma^{\mu \star} F_{\mu \nu} u^{\nu}+\mathrm{M}_{a h} \gamma^{5} \gamma^{\mu} F_{\mu \nu} u^{\nu}
\end{aligned}
$$

In Eq. (14) we use the following notations

$$
\begin{gathered}
R(f)=\sqrt{(f u)^{2}-f^{2}}, \quad s^{\mu}(f)=\frac{u^{\mu}(f u)-f^{\mu}}{\sqrt{(f u)^{2}-f^{2}}} \\
R_{e}=R\left(f^{(e)}\right), \quad R_{\mathrm{N}}=R\left(f^{(\mathrm{N})}\right), \quad s_{(e)}^{\mu}=s^{\mu}\left(f^{(e)}\right), \quad s_{(\mathrm{N})}^{\mu}=s^{\mu}\left(f^{(\mathrm{N})}\right) .
\end{gathered}
$$


When the electromagnetic fields and the potentials of interaction with matter are constant, the solution of Eq. (14) can be written with the use of the resolvent $U(\tau)$

$$
\Psi(\tau)=\frac{1}{\sqrt{2 u_{0}}} U(\tau) \Psi_{0}
$$

where the constant 12-component object $\Psi_{0}$ has the form

$$
\Psi_{0}=\frac{1}{2}\left(1-\gamma^{5} \gamma_{\mu} s_{0}^{\mu}\right)\left(\gamma_{\mu} u^{\mu}+1\right)\left(\psi^{0} \otimes e_{j}\right), \quad \bar{\Psi}_{0} \Psi_{0}=2 .
$$

Here $\psi^{0}$ is a constant bispinor, $e_{j}$ is an arbitrary unit vector in the three-dimensional vector space over the field of complex numbers, and $s_{0}^{\mu}$ is a 4-vector of neutrino polarization such that $\left(u s_{0}\right)=0$. The resolvent in this case takes the form

$$
U(\tau)=e^{-\mathrm{i} \mathcal{F} \tau}
$$

where the matrix $\mathcal{F}$ is defined by Eq. (14).

To calculate the probabilities of transitions between the states with definite flavor and polarization we use the density matrices. Note, that since in our case the states of the neutrino multiplet are pure states, all the results may be obtained with the help of the wave functions, and using density matrices is convenient, though not necessary. The density matrices are defined by the relation

$$
\rho_{\alpha}(\tau)=\frac{1}{4 u^{0}} U(\tau)\left(\gamma^{\mu} u_{\mu}+1\right)\left(1-\gamma^{5} \gamma_{\mu} s_{0}^{\mu}\right) \mathbb{P}_{0}^{(\alpha)} \bar{U}(\tau)=\frac{1}{2 u^{0}} U(\tau)\left(\gamma^{\mu} u_{\mu}+1\right) \mathcal{P}_{0}^{(\alpha)} \bar{U}(\tau)
$$

In this formula $s_{0}^{\mu}$ defines the initial polarization state of the neutrino, and the projector $\mathbb{P}_{0}^{(\alpha)}$ defines its initial flavor state. Thus, $\mathcal{P}_{0}^{(\alpha)}$ is a projector on the initial spin-flavor state of the neutrino. The transition probability from the state $\alpha$ to the state $\beta$ in the time $\tau$ is determined by the following relation

$$
W_{\alpha \rightarrow \beta}=\operatorname{Tr}\left\{\rho_{\alpha}(\tau) \rho_{\beta}^{\dagger}(\tau=0)\right\} .
$$

Even for the constant homogeneous electromagnetic fields and constant potentials of interaction with matter, it is not possible to write an explicit analytical expression for the matrix exponential (19) in the general case. However, we can perform the calculation of the probability, determined by (21) using Backer-Campbell-Hausdorff formula. Then the expression for the transition probability takes the form

$$
W_{\alpha \rightarrow \beta}=\frac{1}{2 u^{0}} \operatorname{Tr}\left\{e^{-\mathrm{i} \tau \mathcal{F}} \mathcal{P}_{0}^{(\alpha)} e^{\mathrm{i} \tau \mathcal{F}} \mathcal{P}_{0}^{(\beta)}\left(\gamma^{\mu} u_{\mu}+1\right) \gamma^{0}\right\}=\frac{1}{2 u^{0}} \sum_{n=0}^{\infty} \frac{(-\mathrm{i} \tau)^{n}}{n !} \operatorname{Tr}\left\{D_{n} \mathcal{P}_{0}^{(\beta)}\left(\gamma^{\mu} u_{\mu}+1\right) \gamma^{0}\right\},
$$

where

$$
D_{0}=\mathcal{P}_{0}^{(\beta)}, D_{1}=\left[\mathcal{F}, \mathcal{P}_{0}^{(\beta)}\right], D_{2}=\left[\mathcal{F},\left[\mathcal{F}, \mathcal{P}_{0}^{(\beta)}\right]\right] \ldots
$$

This series converges well, as it is exponential-type decomposition over the parameter $L / L_{o s c}$, where $L_{o s c}$ is the characteristic oscillation length in vacuum.

\section{Exact solution}

Formula (22) enables one to find the probabilities of different spin-flavor transition probabilities in the case of the constant external conditions. However, if we can find the spin integrals of motion, then the explicit form of the solutions may be obtained. The case when 
neutrino propagates in dense moving unpolarized matter was studied in [14, 15]). Here we will study the case, when neutrino propagates in constant electromagnetic field taking into account transition magnetic moments. The exact solution exists, when the neutrino transition electric moments are neglected.

For simplicity here we consider two-flavor model, where the mass matrix $\mathbb{M}$, the matrix of the transition magnetic moments $\mathrm{M}_{h}$ and the projector on the electron flavor state $\mathrm{P}^{(e)}$ are $2 \times 2$ matrices and the neutrino wave function $\Psi(\tau)$ is an 8-component object. In the mass representation matrices $\mathbb{M}, \mathbb{M}_{h}$ can be expressed in terms of the Pauli matrices as follows

$$
\begin{gathered}
\mathrm{M}=\frac{1}{2}\left(\sigma_{0}\left(m_{1}+m_{2}\right)-\sigma_{3}\left(m_{2}-m_{1}\right)\right), \quad \mathbb{P}^{(e)}=\frac{1}{2}\left(\sigma_{0}+\sigma_{1} \sin 2 \theta+\sigma_{3} \cos 2 \theta\right), \\
\mathbb{M}_{h}=\frac{1}{2}\left(m_{1}+m_{2}\right) \mu_{1} \sigma_{1},
\end{gathered}
$$

where $\sigma_{i}, i=1,2,3$ are the Pauli matrices, $\sigma_{0}$ is the identity $2 \times 2$ matrix, $\theta$ is the vacuum mixing angle.

In the two flavor model for the matrix (14) in the mass representation we have

$$
\mathcal{F} \rightarrow \frac{1}{2}\left\{\left(\sigma_{0}\left(m_{1}+m_{2}\right)-\sigma_{3}\left(m_{2}-m_{1}\right)\right)\left(1-\mu_{0} \gamma^{5} \gamma^{\mu \star} F_{\mu \nu} u^{\nu}\right)-\sigma_{1}\left(m_{1}+m_{2}\right) \mu_{1} \gamma^{5} \gamma^{\mu \star} F_{\mu \nu} u^{\nu}\right\}
$$

The spin integral of motion, which defines the projection of the spin on the direction of the magnetic field in the neutrino rest frame, takes the form

$$
\mathcal{S}=-\gamma^{5} \gamma^{\mu \star} F_{\mu \nu} u^{\nu} / N, \quad N=\sqrt{u_{\mu}^{\star} F^{\mu \alpha \star} F_{\alpha \nu} u^{\nu}} .
$$

To convert the operators, including the resolvent $U(\tau)$, from the mass representation to the flavor representation, one should use the transformation

$$
U(\tau)=\mathbb{U} U^{\prime}(\tau) \mathbb{U}^{\dagger}
$$

where $U^{\prime}(\tau)$ is the resolvent in the mass representation, and $U(\tau)$ is the resolvent in the flavor representation. The Pontecorvo-Maki-Nakagawa-Sakata mixing matrix $\mathbb{U}$ in the two-flavor model is as follows

$$
\mathbb{U}=\left(\begin{array}{cc}
\cos \theta & \sin \theta \\
-\sin \theta & \cos \theta
\end{array}\right) .
$$

The resolvent in the flavor representation is determined by the expression

$$
U(\tau)=\sum_{\zeta= \pm 1} e^{-\mathrm{i} \tau T_{\zeta} / 2}\left(\cos \left(\tau Z_{\zeta} / 2\right)-\mathrm{i}\left(X_{\zeta} \sigma_{1}-Y_{\zeta} \sigma_{3}\right) \sin \left(\tau Z_{\zeta} / 2\right)\right) \Lambda_{\zeta},
$$

where

$$
\begin{aligned}
Y_{\zeta} & =\frac{1}{Z_{\zeta}}\left(\left(m_{2}-m_{1}\right)\left(1-\zeta \mu_{0} N\right) \cos 2 \theta+\zeta \mu_{1} N\left(m_{2}+m_{1}\right) \sin 2 \theta\right) \\
X_{\zeta} & =\frac{1}{Z_{\zeta}}\left(\left(m_{2}-m_{1}\right)\left(1-\zeta \mu_{0} N\right) \sin 2 \theta-\zeta \mu_{1} N\left(m_{2}+m_{1}\right) \cos 2 \theta\right) \\
Z_{\zeta} & =\left\{\left(\left(m_{2}-m_{1}\right)\left(1-\zeta \mu_{0} N\right)\right)^{2}+\left(\left(m_{2}+m_{1}\right) \mu_{1} N\right)^{2}\right\}^{1 / 2} \\
T_{\zeta} & =\left(m_{2}+m_{1}\right)\left(1-\zeta \mu_{0} N\right) .
\end{aligned}
$$

The spin projector has the form

$$
\Lambda_{\zeta}=\frac{1}{2}(1-\zeta \mathcal{S}), \quad\left[\gamma^{\mu} u_{\mu}, \Lambda_{\zeta}\right]=0, \quad \zeta= \pm 1
$$


Now we calculate the spin-flavor transition probabilities. That means, we consider the transitions between the states with definite flavor and helicity. In the flavor representation the projectors on the states with definite flavor take the form

$$
\mathbb{P}_{0}^{(\alpha)}=\frac{1}{2}\left(1+\xi_{0} \sigma_{3}\right), \quad \mathbb{P}_{0}^{(\beta)}=\frac{1}{2}\left(1+\xi_{0}^{\prime} \sigma_{3}\right), \quad \xi_{0}, \xi_{0}^{\prime}= \pm 1
$$

For the projectors on the state with electron flavor one should choose $\xi_{0}, \xi_{0}^{\prime}=1$, otherwise $\xi_{0}, \xi_{0}^{\prime}=-1$. We also assume that in these states neutrino has a definite helicity, i.e.

$$
s_{0}^{(\alpha) \mu}=\zeta_{0} s_{s p}^{\mu}, \quad s_{0}^{(\beta) \mu}=\zeta_{0}^{\prime} s_{s p}^{\mu}, \quad s_{s p}^{\mu}=\left\{|\mathbf{u}|, u^{0} \mathbf{u} /|\mathbf{u}|\right\}, \quad \zeta_{0}, \zeta_{0}^{\prime}= \pm 1 .
$$

To describe neutrino states with left-handed polarization one should choose $\zeta_{0}, \zeta_{0}^{\prime}=-1$, otherwise $\zeta_{0}, \zeta_{0}^{\prime}=1$. Using formula (21), we obtain

$$
\begin{aligned}
& W_{\alpha \rightarrow \beta}=\frac{1+\xi_{0} \xi_{0}^{\prime}}{2} \frac{1+\zeta_{0} \zeta_{0}^{\prime}}{2} W_{1}+\frac{1+\xi_{0} \xi_{0}^{\prime}}{2} \frac{1-\zeta_{0} \zeta_{0}^{\prime}}{2} W_{2} \\
& +\frac{1-\xi_{0} \xi_{0}^{\prime}}{2} \frac{1+\zeta_{0} \zeta_{0}^{\prime}}{2} W_{3}+\frac{1-\xi_{0} \xi_{0}^{\prime}}{2} \frac{1-\zeta_{0} \zeta_{0}^{\prime}}{2} W_{4},
\end{aligned}
$$

where

$$
\begin{aligned}
W_{1}= & \frac{1}{2}\left(\frac{1}{2}\left(1-\zeta_{0}\left(\bar{s} s_{s p}\right)\right)^{2}\left(1-S_{+1}^{2} X_{+1}^{2}\right)+\frac{1}{2}\left(1+\zeta_{0}\left(\bar{s} s_{s p}\right)\right)^{2}\left(1-S_{-1}^{2} X_{-1}^{2}\right)\right. \\
& +\left(1-\left(\bar{s} s_{s p}\right)^{2}\right)\left(C_{+1} C_{-1}+S_{+1} S_{-1} Y_{+1} Y_{-1}\right) \cos (\omega \tau) \\
& \left.+\xi_{0}\left(1-\left(\bar{s} s_{s p}\right)^{2}\right)\left(S_{-1} Y_{-1} C_{+1}-C_{-1} S_{+1} Y_{+1}\right) \sin (\omega \tau)\right), \\
W_{2}= & \frac{1}{2}\left(\frac{1}{2}\left(1-\left(\bar{s} s_{s p}\right)^{2}\right)\left(2-S_{+1}^{2} X_{+1}^{2}-S_{-1}^{2} X_{-1}^{2}\right)\right. \\
& -\left(1-\left(\bar{s} s_{s p}\right)^{2}\right)\left(C_{+1} C_{-1}+S_{+1} S_{-1} Y_{+1} Y_{-1}\right) \cos (\omega \tau) \\
& \left.-\xi_{0}\left(1-\left(\bar{s} s_{s p}\right)^{2}\right)\left(S_{-1} Y_{-1} C_{+1}-C_{-1} S_{+1} Y_{+}\right) \sin (\omega \tau)\right), \\
W_{3}= & \frac{1}{2}\left(\frac{1}{2}\left(1-\zeta_{0}\left(\bar{s} s_{s p}\right)\right)^{2} S_{+1}^{2} X_{+1}^{2}+\frac{1}{2}\left(1+\zeta_{0}\left(\bar{s} s_{s p}\right)\right)^{2} S_{-1}^{2} X_{-1}^{2}\right. \\
& \left.+\left(1-\left(\bar{s} s_{s p}\right)^{2}\right) S_{+1} S_{-1} X_{+1} X_{-1} \cos (\omega \tau)\right), \\
W_{4}= & \frac{1}{2}\left(\frac{1}{2}\left(1-\left(\bar{s} s_{s p}\right)^{2}\right)\left(S_{+1}^{2} X_{+1}^{2}+S_{-1}^{2} X_{-1}^{2}\right)\right. \\
& \left.-\left(1-\left(\bar{s} s_{s p}\right)^{2}\right) S_{+1} S_{-1} X_{+1} X_{-1} \cos (\omega \tau)\right) .
\end{aligned}
$$

Here

$$
\begin{array}{ll}
C_{ \pm 1}=\cos \left(\tau Z_{ \pm 1} / 2\right), & S_{ \pm 1}=\sin \left(\tau Z_{ \pm 1} / 2\right), \\
\omega=\mu_{0}\left(m_{2}+m_{1}\right) N, & \bar{s}^{\mu}=-{ }^{\star} F^{\mu v} u_{v} / N .
\end{array}
$$

The expressions for the probabilities take the same form as those obtained in $[14,15]$ for neutrino in moving matter. The probabilities explicitly depend on neutrino initial flavor $\xi_{0}$ and polarization state $\zeta_{0}$. The probabilities are characterized by six non-multiple frequencies.

By analogy to [14] we can introduce the notations

$$
X_{\zeta}=\sin 2 \theta_{\zeta}=\sin 2\left(\theta_{\zeta}^{m}+\theta\right), \quad Y_{\zeta}=\cos 2 \theta_{\zeta}=\cos 2\left(\theta_{\zeta}^{m}+\theta\right)
$$

Then $\theta_{\zeta}$ can be interpreted as an effective mixing angle for neutrino in electromagnetic field. It is an analog of the famous effective mixing angle in matter [4]. It should be noted that if 
we do not take into account the transition moments, then $\theta_{\zeta}^{m}=0$, and only in this case we can consider a flavor state of the neutrino as a superposition of the mass eigenstates.

As $\sin 2 \theta_{\zeta}$ and $\cos 2 \theta_{\zeta}$ change their sign, when we vary the electromagnetic field (see (31) and (38)), the spin-flavor transition probabilities have resonant character. This resonance is completely analogous to the famous Mikheev-Smirnov-Wolfenstein resonance [5] in matter at rest (the formulas for moving matter were obtained in [14]), though it is caused by completely another physical reason, that is the existence of the transition magnetic moments.

The resonance condition $\cos 2 \theta_{\zeta}=0$ reduces to the relation $\mu_{0} N=1$, if we neglect the ratio $\mu_{1} / \mu_{0}$. When neutrinos propagates orthogonally to magnetic field $\mathbf{B}$, the resonance condition is $u_{0} \mu_{0}|\mathbf{B}|=1$.

\section{Summary}

In conclusion we summarize the main results. We obtain the wave equation for neutrino taking into account neutrino interaction with matter and with external electromagnetic field.

To describe the evolution of ultra-relativistic particles we consider quasi-classical approximation of this equation. We obtain the formal solution of this equation in the case, when the external conditions do not depend on the coordinates of the event space. In this case, using Backer-Campbell-Hausdorff formula we develop a general method of calculating the probabilities of the transitions between arbitrary neutrino spin-flavor states.

We obtain analytical solutions of the quasi-classical neutrino evolution equation and the explicit expressions for the spin-flavor transition probabilities, taking into account the transition magnetic moments. These probabilities take the same form as the spin-flavor transition probabilities for neutrino in moving unpolarized matter, and depend on the initial flavor and polarization state of the neutrino.

We demonstrate, that for neutrino in magnetic field the existence of the transition magnetic moments leads to the resonance behavior of the spin-flavor transition probabilities. This resonance, as well as the Mikheev-Smirnov resonance, takes place when the neutrino state cannot be described as a superposition of the mass eigenstates.

The authors are grateful to A. V. Borisov, A. O. Starinets, I. P. Volobuev and V. Ch. Zhukovskiy for fruitful discussions. A.V. Chukhnova acknowledges the support from the Foundation for the advancement of theoretical physics and mathematics "BASIS".

\section{References}

[1] Pontecorvo B., Zh. Eksp. Teor. Fiz. 33, 549-551 (1957), [Sov. Phys. JETP 6, 429-431 (1958)]

[2] Maki Z., Nakagawa M., Sakata S., Prog. Theor. Phys. 28, 870-880 (1962)

[3] Bilenky S. M., Pontecorvo B., Physics Reports 41, 225-261 (1978)

[4] Wolfenstein L., Phys. Rev. D 17, 2369-2374 (1978)

[5] Mikheev S. P., Smirnov A. Yu., Yad. Phys. 42, 1441-1448 (1985), [Sov. J. Nucl. Phys. 42, 913 (1985)]

[6] Lobanov A. E., Teor. Mat. Fiz. 192, 70-88 (2017), [Theor. Math. Phys. 192, 1000-1015 (2017)]

[7] Lobanov A. E., Ann. Phys. 403, 82-105 (2019), (arXiv: 1507.01256[hep-ph])

[8] Ho C. M., JHEP 2012, 22 (2012)

[9] Bogolubov N. N., Shirkov D. V., Introduction to Theory of Quantized Fields (John Wiley, New York, 1979) 
[10] Lobanov A. E., Izv. Vyssh. Uchebn. Zaved. Fiz. 59, 141-144 (2017), [Russ. Phys. J. 59, 1891-1895 (2017)], (arXiv: 1612.01591[hep-ph])

[11] Chukhnova A. V., Lobanov A. E., arXiv: 1906.09351[hep-ph] (2019)

[12] Giunti C., Studenikin A., Rev. Mod. Phys. 87, 531-591 (2015), (arXiv: 1403.6344[hep$\mathrm{ph}])$

[13] Lobanov A. E., J. Phys. A: Math. Gen. 39, 7517-7529 (2006), (arXiv: hep-ph/0311021)

[14] Lobanov A. E., Chukhnova A. V., Vestn. MGU. Fiz. Astron. 58, No. 5, 22-26 (2017), [Mosc. Univ. Phys. Bull. 72, 454-459 (2017)]

[15] Chukhnova A. V., Lobanov A. E., EPJ Web Conf. 191, 03002 (2018), (arXiv: 1808.07926[hep-ph]) 